\title{
Layered zinc hydroxide-ibuprofen nanohybrids: synthesis and characterization
}

\author{
HAFEZEH NABIPOUR* ${ }^{*}$ and MOAYAD HOSSAINI SADR \\ Department of Chemistry, Faculty of Science, Azarbaijan Shahid Madani University, 53714-161 Tabriz, Iran
}

MS received 6 March 2015; accepted 17 June 2015

\begin{abstract}
The nonsteroidal anti-inflammatory drug (NSAID), ibuprofen (IBU) anion, was intercalated into the layered zinc hydroxide (LZH) to form a new organic-inorganic nanohybrid. Then, IBU-LZH nanohybrid was dispersed into chitosan for the formation of the nanocomposite. The IBU-LZH nanohybrid was characterized by powder X-ray diffraction (PXRD) to study intercalation, scanning electron microscopy (SEM) for the investigation of surface morphology, Fourier transform infrared (FTIR) spectrophotometer to study the chemical interactions and thermal gravimetric analysis/derivative thermogravimetric analysis (TGA/DTG) for understanding the thermal stability. The PXRD patterns showed that the IBU was successfully intercalated into the interlay space of LZHs as monolayers and the basal spacing of LZH increased from 9.57 to $19.54 \AA$. The FTIR analyses confirmed the formation of the host-guest nanohybrid. The DTG studies revealed that the thermal stability of IBU was increased after the intercalation into LZH. The in vitro release study of IBU from IBU-LZH and IBU-LZH/chitosan nanocomposite was investigated in phosphate buffer saline (PBS) solution of pH 4.8 and 7.4. The drug release from the LZH was studied by ultraviolet-visible (UV-vis) spectroscopy.
\end{abstract}

Keywords. Synthesis; ibuprofen; layered zinc hydroxide; nanohybrid.

\section{Introduction}

Organic-inorganic nanohybrids are formed by dispersing inorganic nanostructures in the organic matrices. ${ }^{1}$ The nanohybrids have attracted considerable research interests, due to their unusual physicochemical properties, which cannot be otherwise achieved by any conventional solid state reactions. ${ }^{2}$ This type of material offers a variety of applications in the industries and the environment such as optical and electronic materials, biomaterials, catalysis, sensing, coating, as well as in medical science and energy storage. $^{3-5}$ Layered double hydroxides (LDHs) and layered metal hydroxides (LMHs) have been studied extensively and are recognized for their anion-exchangeable properties. $^{6,7}$ LDHs compounds with inorganic brucitelike structures can be expressed by the general formula $\left[\mathrm{M}(\mathrm{II})_{1-x} \mathrm{M}(\mathrm{III})_{x}(\mathrm{OH})_{2}\right]\left(\mathrm{A}^{n-}\right)_{x / n} \cdot y \mathrm{H}_{2} \mathrm{O}$, where $\mathrm{M}(\mathrm{II})$ is a divalent metal cation $\left(\mathrm{Ca}^{2+}, \mathrm{Mg}^{2+}, \mathrm{Zn}^{2+}, \mathrm{Co}^{2+}, \mathrm{Ni}^{2+}, \mathrm{Cu}^{2+}\right.$ and $\mathrm{Mn}^{2+}$ ) and $\mathrm{M}(\mathrm{III})$ is a trivalent metal cation $\left(\mathrm{Al}^{3+}\right.$, $\mathrm{Cr}^{3+}, \mathrm{Fe}^{3+}, \mathrm{Co}^{3+}, \mathrm{Ni}^{3+}$ and $\left.\mathrm{Mn}^{3+}\right) ; n$ refers to the number of interlayer water molecules, $x$ represents the layer charge density and $\mathrm{A}^{n-}$ refers to the exchangeable anion with a charge $(n-))^{8,9} \mathrm{LZH}$ is a type of $\mathrm{LMH}$, having the empirical formula of $\mathrm{M}(\mathrm{II})(\mathrm{OH})_{2-x}\left(\mathrm{~A}^{n-}\right)_{x / n} \cdot n \mathrm{H}_{2} \mathrm{O}$, where $\mathrm{M}(\mathrm{II})$ is the metal cation, $\mathrm{Zn}^{2+}$ and $\mathrm{A}^{n-}$ are the counterions and the $\mathrm{OH}^{-}$anions on the brucite hydroxide layer are substituted by water molecules and counter anions. ${ }^{10-12}$

*Author for correspondence (ha.nabipour@gmail.com)
LZHs are currently being used as an additive for slow release herbicides and drugs. ${ }^{13-15}$ Some drugs have been intercalated into LZH, including diclofenac sodium, ${ }^{16}$ amoxicillin trihydrate, ${ }^{17}$ ciprofloxacin ${ }^{18}$ and cetirizine. ${ }^{19}$ There are many approaches that can be used to synthesize the LZH nanohybrid such as the co-precipitation method, ${ }^{20}$ thermal decomposition method ${ }^{21}$ and ion-exchange method. ${ }^{22,23}$ From the aforementioned methods, ion-exchange method is frequently chosen for the preparation of LZH nanohybrid because it is an easy method of preparation with fewer steps and lesser involvement of chemicals. ${ }^{24,25}$

NSAIDs are commonly used for the treatment of pain, inflammation, rheumatic disorders and osteoarthritis. ${ }^{26-28}$ Drugs in this category include diclofenac, naproxen, piroxicam, ketorolac, indomethacin, oxaprozin and meloxicam. $\alpha$-Methyl-4-(2-methylpropyl) benzeneacetic acid (ibuprofen) is the oldest of all the innovative NSAIDs available and, in addition, possesses analgesic as well as antipyretic properties $^{29}$ (figure 1). The terminal half-life of ibuprofen is approximately $2 \mathrm{~h}$. According to the Biopharmaceutical Classification System (BCS), IBU had been classified as a class II drug with low solubility at $\mathrm{pH} 1.2$ and 4.5 and high solubility at $\mathrm{pH} 6.8$, consequently, the drug dissolution may be a rate-limiting step in the drug absorption process. ${ }^{30}$ Thus, the improvement of IBU dissolution for its immediate release is desirable for rapid IBU absorption. ${ }^{31}$ In this study, we highlighted the intercalation of IBU into LZH to form a nanohybrid. IBU-LZH was prepared by ion-exchange method under an aqueous condition with the purpose of 
forming a new organic-inorganic nanohybrid for a controlled release formulation. In addition, the effect of the IBU-LZH nanohybrid and IBU-LZH/chitosan nanocomposite on IBU release from $\mathrm{LZH}$ and $\mathrm{LZH} /$ chitosan was also studied.

\section{Methods}

\subsection{Materials}

Ibuprofen was purchased from the Alborz Drug Company in Iran. All other reagents were purchased from Merck Chemical Company and MP Company, and used without further purification.

2.1a Synthesis of LZH: LZH was prepared by the coprecipitation method. Briefly, 0.069 mol of zinc nitrate hexahydrate was dissolved in $20 \mathrm{ml}$ of distilled water. Solutions of $50 \mathrm{ml}$ sodium hydroxide with concentrations of $0.75 \mathrm{~mol} \mathrm{l}^{-1}$ was added into zinc nitrate solution and suspension mixture was stirred for $2 \mathrm{~h}$ at room temperature. A white precipitate was obtained. It was then centrifuged, thoroughly washed with deionized water and dried in an oven for $24 \mathrm{~h}$ at $50^{\circ} \mathrm{C}$.

2.1b Synthesis of IBU-LZH: IBU-LZH was prepared by the ion-exchange method. As described in the earlier works, ${ }^{16}$ about $0.2 \mathrm{~g}$ of LZH was dispersed in $50 \mathrm{ml}$ of decarbonated water, whereas $0.56 \mathrm{~g}$ of IBU was dispersed in $20 \mathrm{ml}$ of distilled water. The two solutions were simultaneously added to a beaker and stirred for $1 \mathrm{~h}$. The $\mathrm{pH}$ of suspension was adjusted to 8 by dropwise addition of $1 \mathrm{~mol}^{-1}$ sodium hydroxide solution. The mixture was stirred for $24 \mathrm{~h}$ at room temperature. The white precipitate was washed free of ions with decarbonated water at least three times, centrifuged at a speed of $1000 \mathrm{rpm}$ for $15 \mathrm{~min}$, and finally dried for $24 \mathrm{~h}$ at $60^{\circ} \mathrm{C}$.

2.1c Synthesis of IBU/chitosan and IBU-LZH/chitosan nanocomposite: A $1 \% \mathrm{v} / \mathrm{v}$ solution of acetic acid was prepared by adding $1 \mathrm{ml}$ of acetic acid to a $100 \mathrm{ml}$ flask half filled with water, which was mixed and then diluted to $100 \mathrm{ml}$. Then, $0.15 \mathrm{~g}$ of chitosan was added to acetic acid solution and stirred for $30 \mathrm{~min}$ at $25^{\circ} \mathrm{C}$ to obtain a transparent liquid. Meanwhile, $0.05 \mathrm{~g}$ of IBU or IBU-LZH was mixed with $100 \mathrm{ml}$ of chitosan dispersion and this suspension was stirred on a magnetic stirrer at room temperature for $24 \mathrm{~h}$.

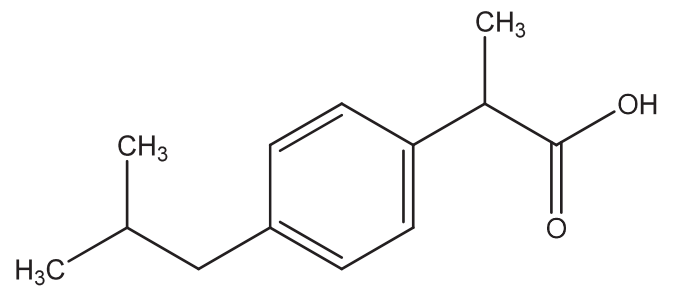

Figure 1. Ibuprofen molecule.
This mixture was added drop by drop into a $1 \% \mathrm{w} / \mathrm{v}$ tripolyphosphate (TPP) solution. The reaction mixture was stirred vigorously and aged for $12 \mathrm{~h}$. Finally, the nanocomposites were washed twice with water and then dried in air.

2.1d Drug-release studies: In-vitro release of IBU from $\mathrm{LZH}$ and $\mathrm{LZH} /$ chitosan was studied at $37^{\circ} \mathrm{C}$ in phosphate buffer saline (PBS) at $\mathrm{pH} 7.4$ and 4.8. Then, $20 \mathrm{mg}$ of the IBU-LZH or IBU-LZH/chitosan was immersed in $10 \mathrm{ml}$ of PBS at $\mathrm{pH} 7.4$ and 4.8 and stirred at $500 \mathrm{rpm}$ at $37^{\circ} \mathrm{C}$ up to $12 \mathrm{~h}$. Every $10 \mathrm{~min}, 3 \mathrm{ml}$ of solution was withdrawn and replaced with fresh phosphate buffer. The amount of IBU release was then measured at $\lambda_{\max } 265 \mathrm{~nm}$ by an UV-vis spectrophotometer.

The per cent of the drug release was evaluated by using the following definitions:

Drug release $(\%)=\frac{\text { Amount of drug release }(\mathrm{mg})}{\text { Total weight of drug sample }(\mathrm{mg})} \times 100$.

\subsection{Characterization}

The powder X-ray diffraction (PXRD) patterns of the samples were collected with a Bruker AXS model D8 advance diffractometer using $\mathrm{CuK}_{\alpha}$ source $(\lambda=1.542 \AA)$ at $40 \mathrm{kV}$, $35 \mathrm{~mA}$, and a scan range $2-70^{\circ}$. Infrared spectra were recorded with a Bruker Fourier transform infrared spectroscopy (FTIR), spectrometer model Bruker, using KBr pellets, scanned from 4000 to $400 \mathrm{~cm}^{-1}$. Absorption spectra were recorded in the range $200-800 \mathrm{~nm}$ on a Shimadzu model 1601 PC UV-vis spectrophotometer. The thermal behaviour of the samples were determined by the thermogravimetric analyses (TGA) on a Mettler-Toledo TGA 851e apparatus in heating rate of $10^{\circ} \mathrm{C} \mathrm{min}^{-1}$ under the $\mathrm{N}_{2}$ atmosphere. Scanning electron microscope (SEM) and micrographs were taken on a VEGA-TESCAN.

\section{Results and discussion}

\subsection{PXRD and structural model}

PXRD is now a common method to study the possibilities of alternations of the LMH structure, after the reaction with the IBU in aqueous solution, and verify the possible changes of the basal spacing after the ion-exchange reaction. PXRD patterns of the pristine LZH, the IBU-LZH nanohybrid and IBU are shown in figure 2. The IBU showed a crystalline structure as previously reported $^{32}$ (figure $2 \mathrm{a}$ ). Figure $2 \mathrm{c}$ exhibits the PXRD patterns of the LZH synthesized by the co-precipitation method. The PXRD patterns are in agreement with the previously published data (JPCDS file no. 01-72-0627, $\left.\mathrm{Zn}_{5}(\mathrm{OH})_{8}\left(\mathrm{NO}_{3}\right)_{2} \cdot 2 \mathrm{H}_{2} \mathrm{O}\right)$ and showed high crystallinity of $9.57 \AA$ with basal spacing, due to the 200 planes of the monoclinic structure. ${ }^{5}$ The XRD pattern of IBU intercalated into $\mathrm{LZH}$ is shown in figure $2 \mathrm{~b}$ that exhibited the characteristic reflections of a layered nanohybrid which are 


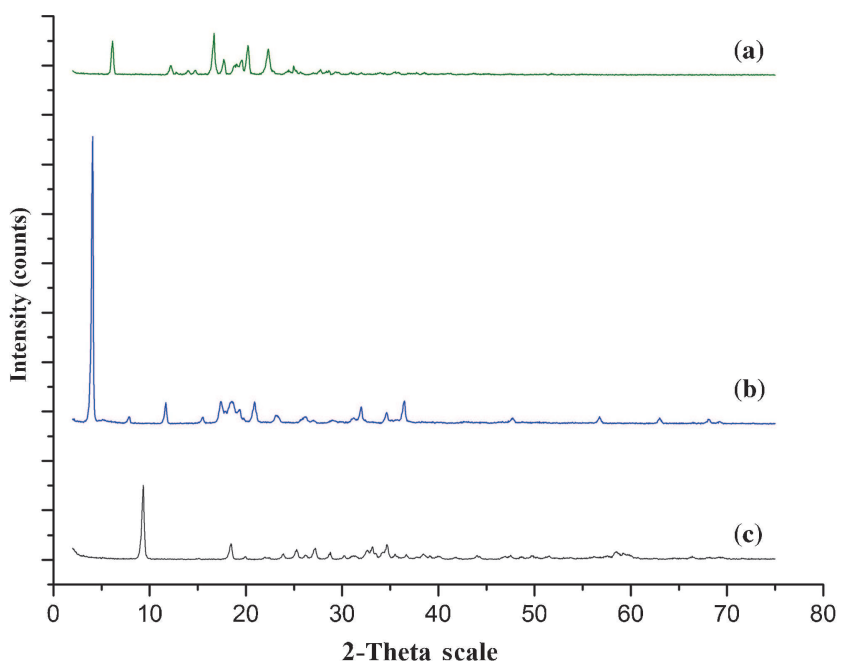

Figure 2. Powder X-ray diffraction patterns of (a) IBU, (b) IBU$\mathrm{LZH}$ and (c) LZH.

sharp at lower theta angle and weaker at higher angles. The shifting of basal reflection towards low $2 \theta$ angle $\left(2 \theta=3.70^{\circ}\right.$, due to a $d$-spacing of $19.54 \AA$ ) for the IBU-LZH confirms the intercalation of IBU into the interlayer galleries of the LZH.

The 3D molecular size, along with the $x, y$ and $z$ axes of IBU was estimated to be $9.54,4.89$ and $2.4 \AA$ by ChemOffice software (Cambridge, MA, USA). Assuming a thickness of $4.8 \AA$ for the LZH layer and $2.6 \AA$ for each zinc tetrahedra, the gallery height for LZH nanohybrid was estimated to be $19.54 \AA$. Therefore, the gallery height of the LZH after the intercalation of IBU could be calculated by subtracting the thickness of the LZH layer plus the height of zinc cation moiety of the lattice from $d$-spacing, and found to be $9.54 \AA$ $(19.54-(4.8+2.6+2.6) \AA)$. The obtained value $(9.54 \AA)$ was larger than the value of long and short axes of IBU molecule (figure 3a). Therefore, this suggests that IBU anions were arranged as monolayers in the interlayer of $\mathrm{LZH}^{33}$ Figure $3 \mathrm{~b}$ shows IBU anions intercalated in the interlayer space.

\subsection{FTIR}

FTIR can be very helpful to show the successful intercalation of the anions into LZH. The FTIR for pristine IBU, IBU intercalated into the LZH interlayer and LZH are shown in figure 4. FTIR spectra of LZH showed two peaks at 438 and $468 \mathrm{~cm}^{-1}$, respectively, due to the vibration of zinc-oxygen bonds. The absorption at $641 \mathrm{~cm}^{-1}$ can be assigned to the stretching vibration of oxygen-hydrogen. The weak absorption at $833 \mathrm{~cm}^{-1}$ is due to $v_{2}$ mode of nitrate anions. The sharp absorption at $1373 \mathrm{~cm}^{-1}$ is due to $v_{3}$ vibrational mode of nitrate ion with $\mathrm{D}_{3}$ h symmetry and ions intercalated in the interlayer spacing. An absorption band at $1640 \mathrm{~cm}^{-1}$ is attributed to $\delta$-mode of oxygen-hydrogen groups. The $3486 \mathrm{~cm}^{-1}$ band due to hydroxyl groups of water molecules is placed in among the layers or is physisorbed onto the layer. An absorption band at around $3580 \mathrm{~cm}^{-1}$ is attributed to

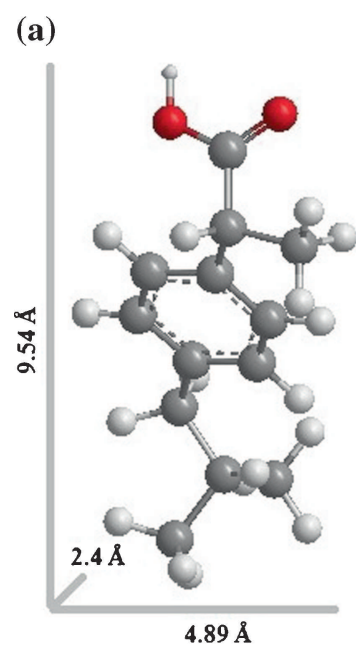

(b)

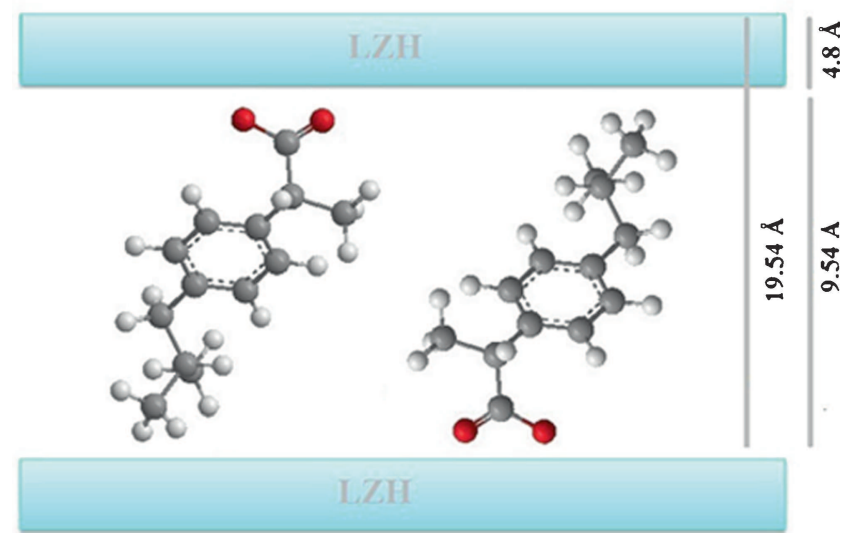

Figure 3. Molecular structure of IBU and 3D molecular size of (a) IBU and (b) spatial orientation of IBU after intercalation.

stretching vibrations of hydroxyl groups on lattice ${ }^{17}$ (figure $4 c)$. Figure $4 \mathrm{~b}$ shows the FTIR spectra used to prove the intercalation of IBU into the LZH host. A very broad absorption band at about $3354 \mathrm{~cm}^{-1}$ is related to the $v(\mathrm{OH})$ stretching vibration of the hydroxyl group of the host layers as well as interlayer and physically adsorbed water molecules. Typical $v(\mathrm{CH})$ stretching vibrations of nanohybrid are observed at 2953, 2931 and $2868 \mathrm{~cm}^{-1}$. The absorption bands at 1549 and $1366 \mathrm{~cm}^{-1}$ are related to the asymmetric and symmetric stretching of $\mathrm{COO}^{-}$group, respectively. The $\mathrm{C}=\mathrm{O}$ stretching vibration of the $-\mathrm{COO}^{-}$group at $1721 \mathrm{~cm}^{-1}$ vanished in IBU. A band at $718 \mathrm{~cm}^{-1}$ may be attributed to the hydroxyl bending within the layers. The peaks and bonds at 422 and $480 \mathrm{~cm}^{-1}$ are due to the $\mathrm{Zn}-\mathrm{OH}$ and $\mathrm{Zn}-\mathrm{O}$ lattice vibrations. Figure 4a displays the FTIR spectra of IBU. The peak at 2956 and $2869 \mathrm{~cm}^{-1}$ is attributed to $\mathrm{CH}_{3}$ asymmetric stretching and $\mathrm{CH}_{2}$ asymmetric stretching vibration, respectively.

The band at $1721 \mathrm{~cm}^{-1}$ is due to asymmetrical stretching vibration of the $-\mathrm{COOH}$ group. The sharp peaks at 1508 and $1420 \mathrm{~cm}^{-1}$ corresponded to $\mathrm{C}-\mathrm{C}$ stretching vibration in the benzene ring. An absorption band at $1231 \mathrm{~cm}^{-1}$ is due to $\mathrm{C}-\mathrm{C}$ 


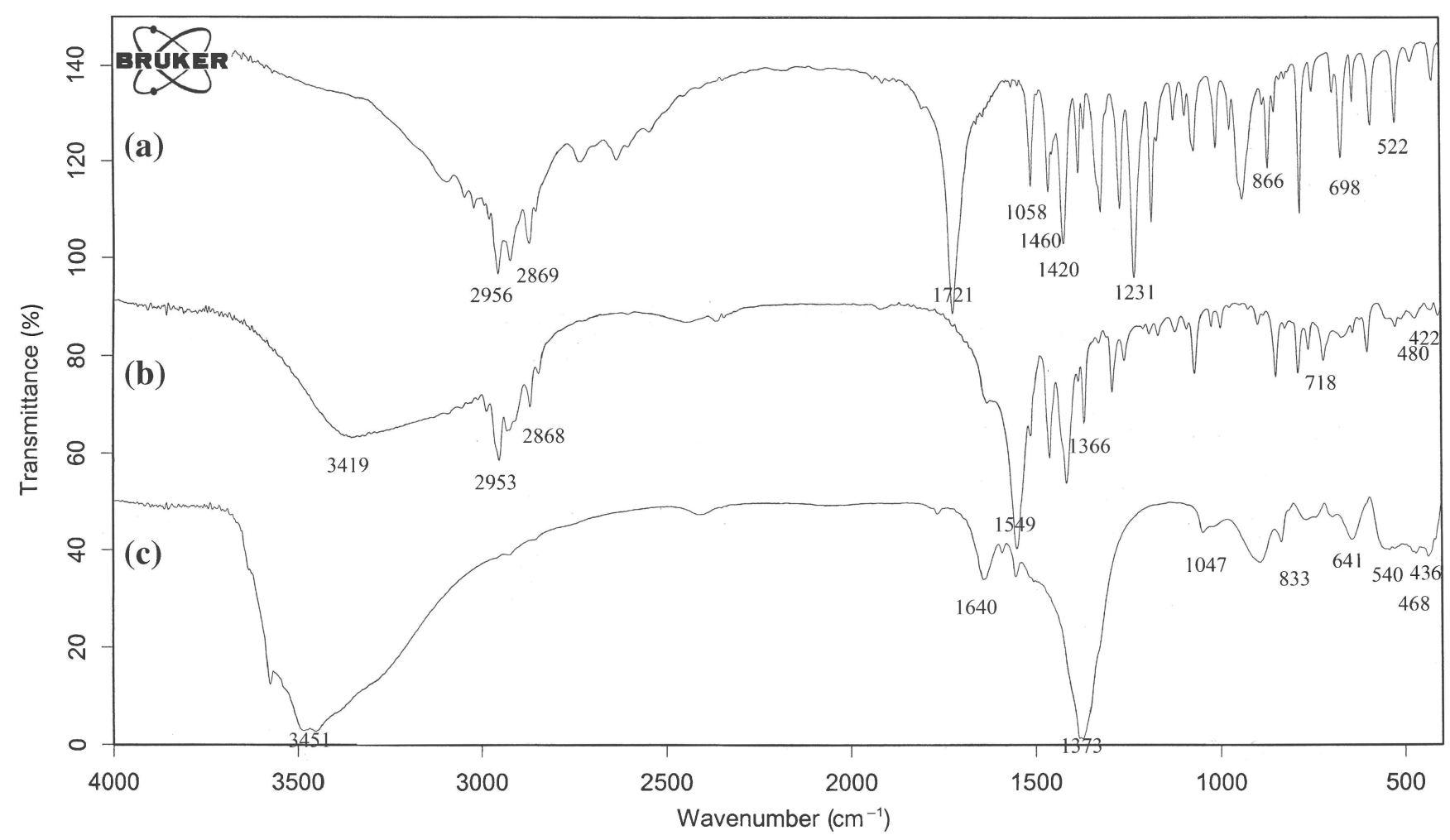

Figure 4. FTIR spectra of (a) IBU, (b) IBU-LZH and (c) LZH.

stretching vibration. Bands centred at 1460, 866, 407 and $522 \mathrm{~cm}^{-1}$ are assigned to $\mathrm{CH}_{3}$ asymmetric deformation, $\mathrm{C}-\mathrm{H}$ out of plane deformation, in plane ring deformation and $\mathrm{CH}_{2}$ inplane rocking vibration, respectively. ${ }^{34}$ The FTIR results confirmed the presence of IBU anions between the layers of LZH.

\subsection{Thermal study}

The curves of IBU and IBU-LZH are presented in figure 5. For IBU (figure 5a), thermal studies show that the temperature maxima is at $202.2^{\circ} \mathrm{C}$ with weight loss of $99.8 \%$ compared to $409.3^{\circ} \mathrm{C}$ for the IBU-LZH with weight loss of $78 \%$. This indicates that IBU encapsulated into the inorganic interlamellae is thermally more stable than their counterpart in the anion form. DTG of IBU-LZH showed (figure 5b) a peak at $69.2^{\circ} \mathrm{C}$ with weight losses of $3.0 \%$ due to the removal of surface physisorbed and intercalated structure water molecules. The second weight loss at $409.3^{\circ} \mathrm{C}$ is due to dehydroxylation of the hydroxyl layer together with the decomposition of IBU. Increasing the decomposition temperature of IBU from $202.2^{\circ} \mathrm{C}$ for IUB pure to $409.3^{\circ} \mathrm{C}$ for IBU-LZH nanohybrid confirmed the greater thermal stability of IBU in nanohybrid than their counterpart in the unbound compound form.

\subsection{Surface morphology study}

The surface morphologies of LZH and IBU-LZH are shown in figure 6. The SEM micrograph of LZH in figure 6a
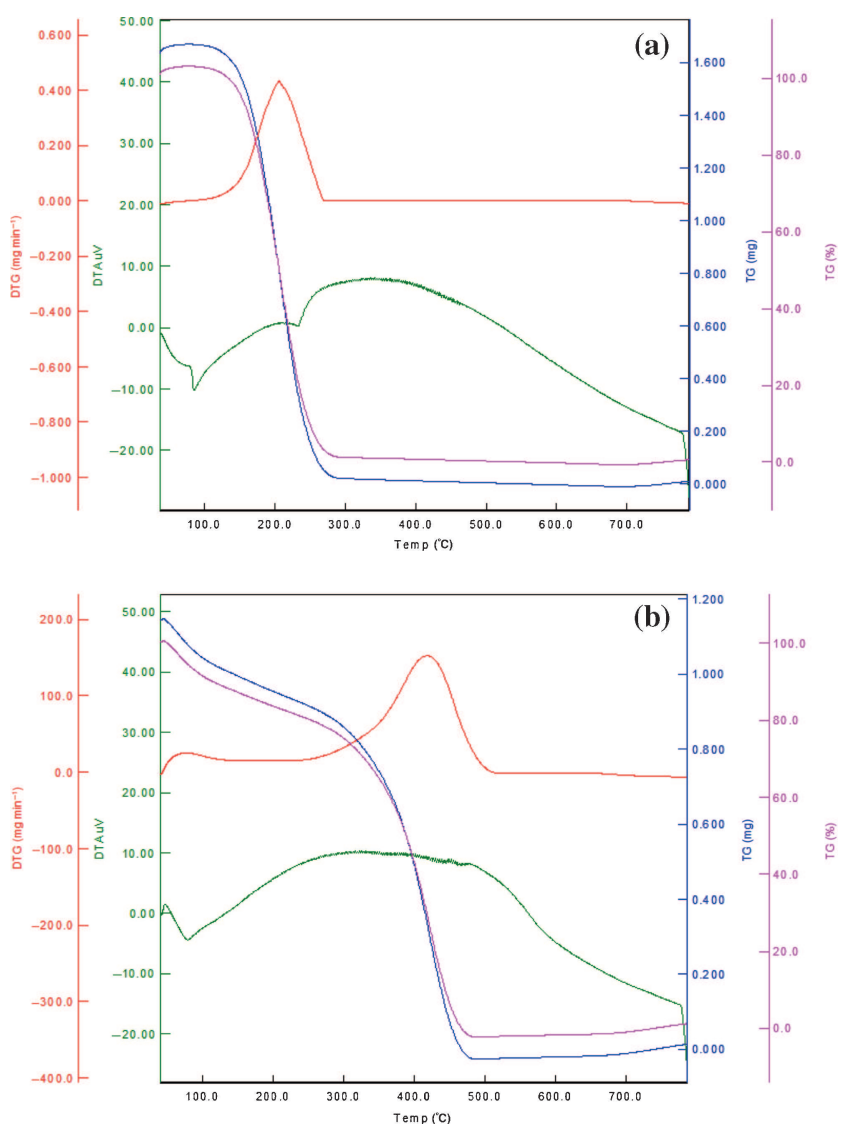

Figure 5. TGA/DTG thermograms of (a) IBU and (b) IBU-LZH nanohybrid. 

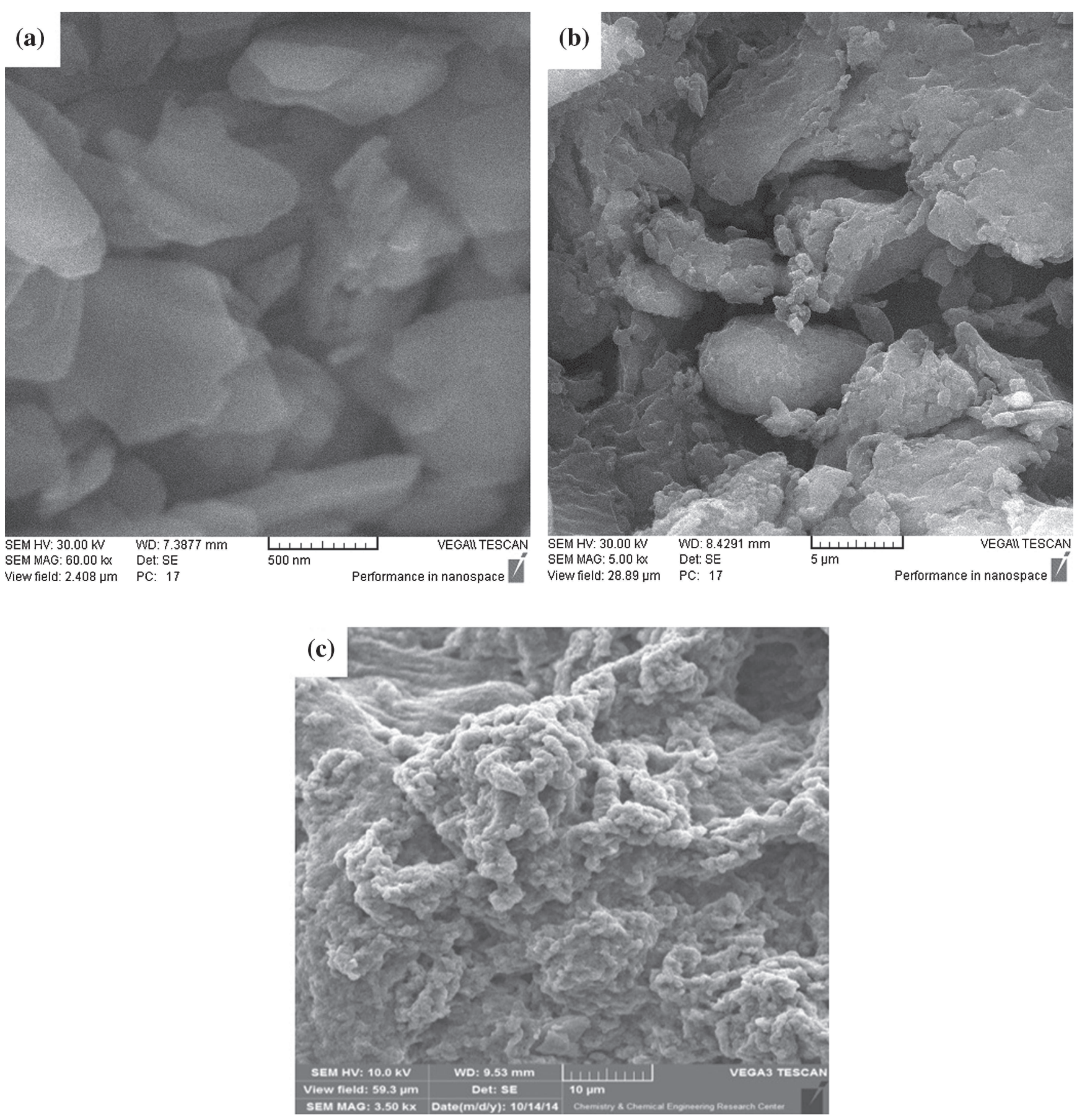

Figure 6. SEM images of (a) LZH, (b) IBU-LZH and (c) IBU-LZH/chitosan.

displayed aggregated flake-like particles. The IBU-LZH nanohybrid showed compact irregular agglomerated structure with non-uniform size (figure 6b). These images exhibited that the transformation of LZH into an IBU-LZH nanohybrid produced surface morphology transformation and the particle size of IBU-LZH nanohybrid was reduced in comparison with the layered hydroxide. ${ }^{16}$ This indicated that the IBU anions intercalated into the interlayer space of LZH. Figure $6 \mathrm{c}$ shows a non-uniform structure for IBULZH/chitosan.

\subsection{The release behaviour of IBU from nanohybrid and} nanocomposite

The release profiles of IBU from chitosan, IBU-LZH and IBU-LZH/chitosan in PBS solution are displayed in figure 7. The release rate of IBU from chitosan, nanohybrid and nanocomposite is clearly defendant on the $\mathrm{pH}$ values; i.e., release of IBU at $\mathrm{pH} 7.4$ is slower than that at $\mathrm{pH} 4.8$, where the amount of drug released reaches $77 \%$ in 1200 min (figure 7a). The sustained release properties of IBU anions from 

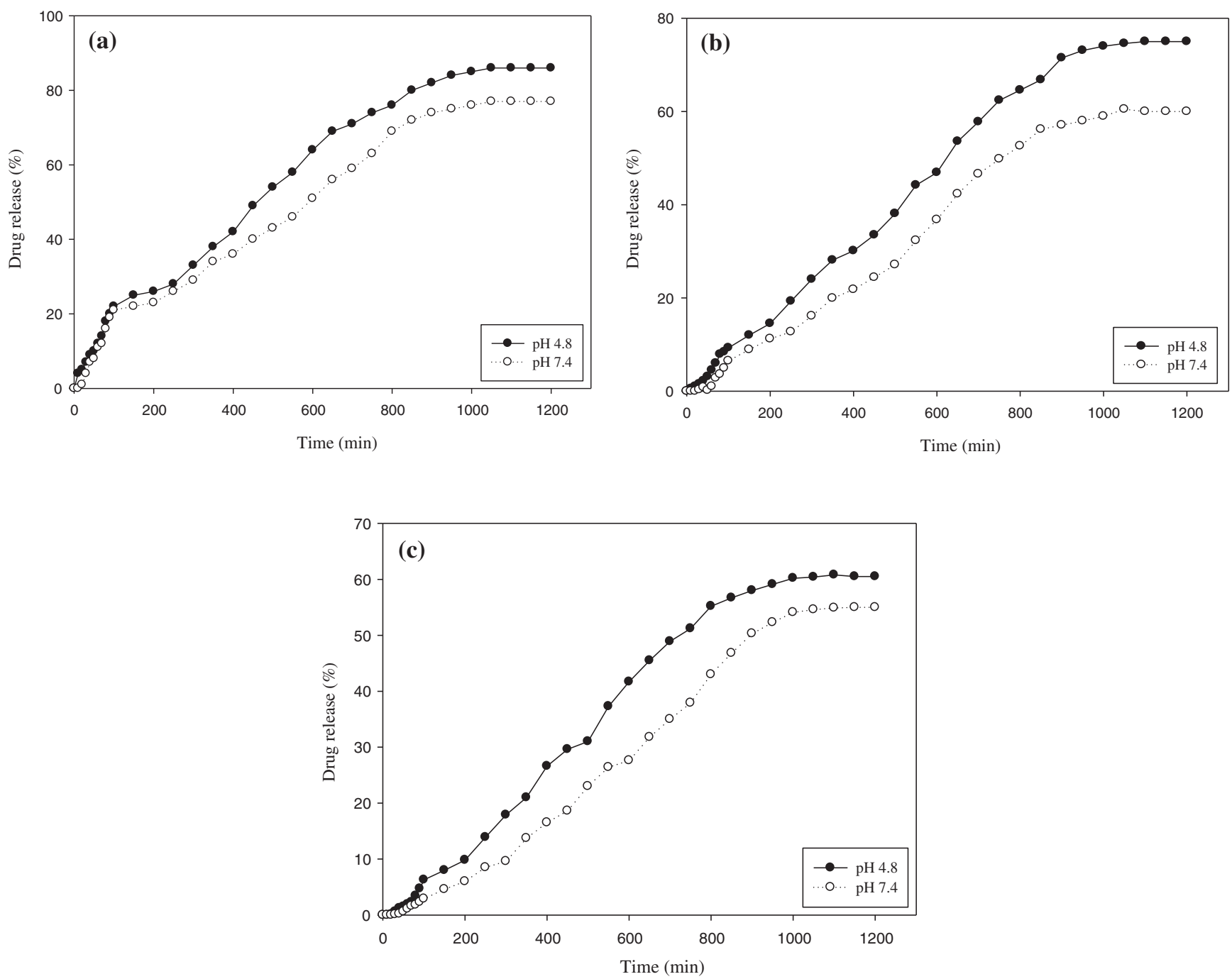

Figure 7. Release properties of IBU from (a) LZH, (b) chitosan and (c) LZH-chitosan nanocomposite at pH 4.8 and 7.4.

the nanohybrids were related to strong electrostatic interaction between the positively charged layered hydroxide and the negatively charged IBU anions in the interlayer. The increased release rate of IBU in $\mathrm{pH} 4.8$ medium was noted. The difference in release rates may be due to the solubility of IBU in release media of different $\mathrm{pH}$ values. IBU is practically insoluble in acidic $\mathrm{pH}$ but is readily soluble in alkaline $\mathrm{pH} .{ }^{35}$ Figure $7 \mathrm{~b}$ shows a release of IBU from IBUchitosan nanohybrid at $\mathrm{pH} 7.4$ and 4.8. The release rates of IBU from IBU-LZH at $\mathrm{pH} 7.4$ is slower than the release rate of IBU at $\mathrm{pH} 4.8$, where the release is $>75 \%$ at $\mathrm{pH} 7.4$ for 1200 min compared to the release of $>60 \%$ at $\mathrm{pH} 4.8$. This is due to the amine groups of chitosan that gets protonated, leading to the dissociation of the hydrogen bonds involving the chitosan amine groups with the carboxylic acid groups of the IBU molecules. The release behaviours at $\mathrm{pH} 4.8$ in IBU-LZH/chitosan were very fast compared to $\mathrm{pH} 7.4$, which can be attributed to the chitosan matrix. However, chitosan could not cap the openings of LZH. Consequently, the IBU anions can easily diffuse out from the LZH interlayer into the release medium.

\section{Conclusion}

The NSAID molecule IBU has been included within the galleries of the anionic clay, LZH by ion-exchange intercalation. PXRD, FTIR spectroscopy, SEM and DTA/DTG analysis have been used to characterize the confined IBU anions in the LZH drug nanohybrids.

PXRD suggested an expansion of interlayer spacing to $19.54 \AA$ due to the monolayer arrangement of IBU anions. The FTIR analyses indicated a successful intercalation of IBU anions between the LZH layers. Surface area analysis displays the increase in surface area dramatically after intercalation and TGA/DTG analysis exhibits the obviously improved thermal stability of intercalated IBU anions after intercalating into LZH interlayer due to the host-guest interaction. The particle size of IBU anions intercalated LZHs was $50-100 \mathrm{~nm}$. The strong electrostatic intercalations between the LZH layers and the organic species exhibited the controlled release of IBU anions in the interlayer. The results of in vitro drug release from the nanohybrid and nanocomposite indicated that the release rate from 
nanocomposite is remarkably lower than that from the IBUchitosan and nanohybrid at $\mathrm{pH}$ 7.4. These results show that layered zinc hydroxide may have application as the basis of a drug delivery system.

\section{Acknowledgements}

The financial support from Azarbaijan Shahid Madani University Research Council and International Imam Khomeini University is greatly appreciated.

\section{References}

1. Sanchez C, Julian B, Belleville P and Popall M 2005 J. Mater. Chem. 153559

2. Choy J H, Kwon S J and Park G S 1998 Science 2801589

3. Hussein M Z, Ghotbi M Y, Yahaya A H and Rahman M Z A 2009 Mater. Chem. Phys. 113491

4. Kasai A and Fujihara S 2006 Inorg. Chem. 45415

5. Yeganeh Ghotbi M, Bagheri N and Sadrnezhaad S K $2011 J$. Colloid. Interface Sci. 36289

6. Newman S P and Jones W 1999 J. Solid State Chem. 14826

7. Morioka H, Tagaya H, Kasaru M, Kadokawa J and Chiba K 1999 Inorg. Chem. 384211

8. Seida Y and Nakano Y 2002 Water Res. 361306

9. Kwon S J and Choy J H 2003 Inorg. Chem. 428134

10. Altuntasoglu O, Matsuda Y, Ida S and Matsumoto Y 2010 Chem. Mater. 223158

11. Zhao L, Miao J, Wang H, Ishikawa Y and Feng Q 2011 Chin. J. Chem. 291837

12. Arizaga G G C, Satyanarayana K G and Wypych F 2007 Solid State Ion. 1781143

13. Barahuie F, Hussein M Z, Arulselvan P, Fakurazi S and Zainal Z 2013 Sci. Adv. Mater. 51983

14. Choy J H, Shin J, Lim S Y, Oh J M, Oh M H and Oh H 2010 J. Food Sci. 75 N63
15. Hussein M Z, Al Ali S H, Zainal Z and Hakim M N 2011 Int. J. Nanomed. 61373

16. Nabipour H and Hosaini Sadr M 2015 J. Porous Mater. 22447

17. Nabipour H, Hosaini Sadr M and Thomas N 2015 J. Exp. Nanosci. doi: 10.1080/17458080.2014.998301

18. Latip A F A, Hussein M Z, Stanslas J, Choon W C and Adnan R 2013 Chem. Cent. J. 7119

19. Barahuie F, Hussein M Z, Abd Gani S H, Fakurazi S H and Zainal Z 2014 Int. J. Nanomed. 93137

20. He J, Wei M, Li B, Kang Y, Evans D G and Duan X 2006 Struct. Bond. 11989

21. Machovsky M, Kuritka K I, Sedlak J and Pastorek M 2013 Mater. Res. Bull. 484002

22. Ishikawa T, Matsumoto K, Kandori K and Nakayama T 2007 Colloids Surf. A: Physicochem. Eng. Asp. 293135

23. Biswick T, Park D H and Choy J H 2012 J. Phys. Chem. Solids 731510

24. Bashi A M, Hussein M Z, Zainal Z and Tichit D 2013 J. Solid State Chem. 20319

25. Bashi A M, Haddawi S M and Mezaal M A 2013 Arab. J. Sci. Eng. 381663

26. Regula J, Butruk E, Dekkers C P, de Boer S Y, Raps D, Simon L, Terjung A, Thomas K B, Lühmann R and Fischer R 2006 Am. J. Gastroenterol. 1011747

27. Thompson M and Percy J S 1966 Br. Med. J. 180

28. Harth M and Bondy D C 1969 Can. Med. Assoc. J. 101311

29. Roth S H 1985 Rheumatic therapeutics (New York: McGrawHill)

30. Amidon G L, Lennernäs H, Shah V P and Crison J R 1995 Pharm. Res. 12413

31. Xu L, Li S M and Sunada H 2007 Chem. Pharm. Bull. 551545

32. Patrick Stahly G, McKenzie A T, Andres M C, Russell C A, Byrn S R and Johnson P 1997 J. Pharm. Sci. 86970

33. Gunawan P and Xu R 2008 J. Pharm. Sci. 974367

34. Ramukutty S and Ramachandran E 2012 Cryst. Res. Technol. 4731

35. Depan D, Saikia L and Singh P 2010 Macromol. Symp. 28780 